\title{
Radar observations in the vicinity of pre-noon auroral arcs
}

\author{
H. Nilsson ${ }^{1}$, A. Kozlovsky ${ }^{2,3}$, T. Sergienko ${ }^{1}$, and A. Kotikov ${ }^{4}$ \\ ${ }^{1}$ Swedish Institute of Space Physics, Kiruna, Sweden \\ ${ }^{2}$ Oulu University, Oulu, Finland \\ ${ }^{3}$ Sodankylä Geophysical Observatory, Sodankylä, Finland \\ ${ }^{4}$ St. Petersburg State University, St. Petersburg, Russia
}

Received: 5 January 2005 - Revised: 3 May 2005 - Accepted: 17 May 2005 - Published: 28 July 2005

\begin{abstract}
A combination of EISCAT incoherent scatter radar measurements, optical and magnetometer data is used to study the plasma in and around pre-noon structured precipitation and auroral arcs. Particular attention is paid to regions of comparatively low E-region density observed adjacent to arcs or structured precipitation in the EISCAT Svalbard radar field-aligned measurements. Comparison between luminosity and incoherent scatter electron density measurements shows that the low-density regions occur primarily due to the absence of diffuse precipitation rather than to a cavity formation process. Two cases of arcs and low density/luminosity regions are identified. The first is related to a strong Pc5 pulsation event, and the absence of diffuse precipitation is due to a large-scale modulation of the diffuse precipitation. In the second case the equatormost arc is on a shielding boundary and the low-density region coincides with a strong flow region just poleward of this arc. Regions of high electric field and low luminosity and conductance are observed prior to intensification of the structured precipitation in both cases. The ionospheric current is enhanced in the low conductance region, indicating that the strong electric fields do not result solely from ionospheric polarization electric fields, and thus are mainly driven by magnetospheric processes. The average energy of the precipitating electrons in the arcs and structured precipitation is, according to EISCAT measurements, $500 \mathrm{eV}$ and the energy spectra are similar for the pulsation and shielding cases. The average energy is thus significantly less than in the diffuse precipitation region which shows central CPS-like energy spectra. We suggest that the low ionospheric conductance of $0.7 \mathrm{~S}$ in the low density regions is favorable for the arc formation process. This is in quantitative agreement with recent simulations of the ionospheric feedback instability.
\end{abstract}

Keywords. Magnetospheric physics (Auroral phenomena; Magnetosphere-ionosphere interactions) - Ionosphere (Plasma convection)

Correspondence to: H. Nilsson

(hans.nilsson@irf.se)

\section{Introduction}

The main objective of this paper is to study the characteristics of the plasma in the vicinity of dayside auroral arcs by means of field-aligned incoherent scatter radar data. Particular attention is paid to the conductance, as this is a key factor determining whether the ionosphere may play an active role in the arc formation process through ionospheric feedback (e.g. Lysak and Song, 2002). While many detailed studies have estimated the conductance in and around nightside auroral arcs (see Aikio et al., 2002, for a recent paper), there are no reports of such measurements in and around dayside aurora. There are two main reasons for this: (1) The scarcity of data, as it is only the EISCAT Svalbard radar which can yield conjugate optical and incoherent scatter radar data from dayside auroral arcs, and this only for a few weeks per year during the polar night; and (2) most attention has been paid to the cusp-related aurora where estimates of plasma flow are considered more important than conductance estimates, so that the radar mode used usually does not yield useful conductance estimates (i.e. low elevation measurements are often used to observe line-of-sight velocities, rather than magnetic field-aligned measurements, e.g. Moen et al., 2001). Even when the radar has been operated in a field-aligned position, no attention has so far been paid to conductance estimates (e.g. McCrea et al., 2000; Lockwood et al., 2000).

The fact that the background conductance plays a role for the large-scale distribution of auroral acceleration events is well-established (e.g. Newell et al., 1996). If this is due to an ionospheric feedback instability, then low background conductance will also be favorable for electron acceleration on smaller spatial and temporal scales (e.g. Lysak and Song, 2002; Pokhotelov et al., 2002a,b) than the diurnal variations determined by Newell et al. (1996). In general, ionospheric conductance must affect arc formation directly on the arc formation time scale, so that if it is important for the summerwinter asymmetry, as suggested in the previously cited reports, it must be important for single events, as well. The level of the background conductance can also be important for the distribution of field-line oscillations, as low enough 
conductance can reduce the ionospheric reflectivity and thus strongly dampen the imposed oscillations (Lysak, 1990). As such oscillations may be associated with aurora (e.g. Samson et al., 1991; Kozlovsky and Kangas, 2002) this may in turn influence the presence of aurora. Furthermore, the ionospheric conductance may affect the threshold for the interchange instability, which is another mechanism proposed for auroral arc formation (e.g. Golovchanskaya and Maltsev, 2003). In general terms, high ionospheric conductance will have a damping effect on any magnetospheric instability which depends on large electric fields developing during the course of the instability, as these may be short-circuited through the ionosphere.

Magnetospheric current systems may not only create arcs and associated conductance enhancements. The cause and effect may be the opposite, as discussed above, with downward current systems associated with magnetospheric processes causing low density and thus low conductance regions in the ionosphere. This phenomenon, "E-region cavity formation", has been modeled by Banks and Yasuhara (1978); Doe et al. (1995); Karlsson and Marklund (1998), and cavity formation against a solar EUV produced E-region background in the dayside cusp region was observed by Nilsson et al. (1998). Such cavity formation may in turn contribute to further ionospheric feedback, as modeled by Streltsov and Lotko (2002).

Even though ionospheric conductance may be important, it will only provide a modification of a magnetospheric energy input. This energy input can essentially come from either the dayside (direct solar wind-magnetosphere interaction, mainly through the cusp) or the nightside (i.e. the dayside aurora can be regarded as an extension towards midday local time of the nightside auroral oval). An aim of all descriptions and classifications of auroral arcs in the dayside is to distinguish between these two major sources. The commonly used arc classification of Sandholt et al. (1998) describes and classifies all kinds of dayside aurora in relation to the interplanetary magnetic field (IMF), as at least the location may be affected by the IMF even if the aurora occur on closed field-lines. We will relate to the classification scheme of Sandholt et al. (1998) when we discuss our arc observations.

Most published studies on dayside aurora concern poleward-moving auroral forms (PMAF) on open magnetic field-lines, closely linked to the reconnection of magnetospheric field-lines to the solar wind (e.g. Sandholt et al., 1996; Fasel, 1995). However, the closed field-line dayside region is also rich in auroral phenomena, as reported in a number of papers. We will just mention a few of particular relevance to this study here: Milan et al. (1999) reported short-period (1-2 min), poleward-moving auroral forms similar to the cusp-related auroral forms, but apparently related to the post-noon convection reversal. It was thus proposed that this aurora was the optical signature of boundary plasma sheet precipitation in the region 1 field-aligned current system. The periodicity was attributed to ULF wave activity. Prikryl et al. (1999) showed evidence that subcusp ULF wave activity in the form of Pc5 pulsations was driven by changes in the solar wind and could thus be very similar to phenomena on open field-lines. It was even suggested that field-line resonances occurring just equatorward of the cusp could lead to a reverse feedback, modulating the reconnection rate. Kozlovsky and Kangas (2002) explained poleward moving auroral forms on closed field-lines in terms of field-line eigenmode oscillations. Another suggested that a source process for dayside arcs is the interchange instability (e.g. Kozlovsky et al., 2003; Safargaleev et al., 2003). The interchange instability is expected to lead to current sheet splitting, and upward current regions should be favorable for arc formation. At the inner plasma sheet boundary the same mechanism leads to a stability, deflecting the plasma from reaching lower latitudes. This shielding effect leads to the formation of the Alfvén layer and region 2 field-aligned current (e.g. Blanc and Caudal, 1985), which is upward in the morning auroral oval and thus favorable for arc formation.

In this study we complement previous studies using joint optical (all-sky camera), convection (EISCAT VHF radar at low elevation, IMAGE magnetometer chain) and EISCAT Svalbard field-aligned measurements in the vicinity of prenoon arcs, obtained during the polar night on 21 December 1998. The data set allows us to determine the geophysical region in which the aurora occur, as well as the electric fields and current systems associated with the arcs. Furthermore, we can study the relation between ionospheric conductance and pre-noon auroral arcs, including the possible role of background conductance in the arc formation process, as well as the role of the arc current system, in creating structure in the ionospheric conductance.

\section{Instruments}

For the present study we have used data from the EISCAT Svalbard radar (ESR), the EISCAT VHF radar located in Troms $\varnothing$, the Finnish Meteorological Institute all-sky camera in Longyearbyen, the IMAGE magnetometer chain, the Wind and IMP-8 solar wind satellites and data from the Polar satellite.

The EISCAT Svalbard radar is located near Longyearbyen, at $78.153^{\circ} \mathrm{N}, 16.029^{\circ} \mathrm{E}$, corrected geomagnetic coordinates $75.19^{\circ} \mathrm{N}, 112.13^{\circ} \mathrm{E}$. The radar was operated in the GUP3 mode, providing estimates of electron density, electron and ion temperature and line-of-sight velocity. The radar beam was directed along the geomagnetic field, using $81.6^{\circ}$ elevation and $180.6^{\circ}$ azimuth ( 0 to the north, positive clockwise). Altitude profiles of E-region densities allow for an estimate of ionization rates (due to the low life time of density enhancements at these altitudes, though an estimate of the time derivative of electron density or steady state assumption must be used), and can thus serve to indicate whether an auroral arc caused by emissions from the E-region was within the radar beam. Precipitation may also enhance electron temperatures, in particular the F-region electron temperature responds quickly and strongly to soft electron precipitation 
$(100 \mathrm{eV})$, as, for example, expected within the ionospheric projection of the cusp (Lockwood et al., 1993; Watermann et al., 1994; Nilsson et al., 1994). Ion temperatures are significantly enhanced by Joule heating in the presence of enhanced electric fields, so that ion temperature enhancements can be used to obtain a morphological picture of the presence of enhanced electric fields. Using modeling work such as that of Schunk et al. (1975), it is also possible to obtain a rough estimate of the magnitude of the electric field enhancement (ignoring any influence of the neutral wind, which must be assumed to be insignificant).

Ionospheric plasma drift (ion velocity) in the F-region over Svalbard was observed using the EISCAT-VHF common program $\mathrm{CP}-4 \mathrm{~B}$ experiment. The radar is located in Troms $\varnothing\left(69.58^{\circ} \mathrm{N}, 19.21^{\circ} \mathrm{E}\right)$, but the $\mathrm{CP} 4$ program allows one to reach higher latitudes, approaching $80^{\circ}$. It uses the VHF radar in split-beam mode to measure at an elevation of $30^{\circ}$ to the horizon both towards the geographic north and somewhat to the west simultaneously. For both beams, data was obtained from twenty scattering volumes (gates), $40 \mathrm{~km}$ apart, with a temporal resolution of $1 \mathrm{~min}$. Vectors of the plasma velocity were calculated for a given geomagnetic latitude by combining the line-of-sight velocities from the two beams and using the beam-swinging method (see Kozlovsky and Kangas, 2002, for more details). East-west flow estimates are obtained from the difference between the two radar beams, i.e. longitudinal homogeneity is assumed. The northsouth estimates are thus much more reliable. Estimates of the ion temperature can also be used to infer high drift velocity. Further support for the convection studies can be obtained through magnetic field data from the Ny Ålesund (NAL), Longyearbyen (LYR), Hornsund (HOR), Hopen Island (HOP), and Bear Island (BJN) stations of the IMAGE magnetometer network. By assuming mainly east-west currents (magnetometer X-component), one may also utilize the information from the Z-component and the combined magnetometer data from the chain to obtain the structure of the currents in more detail through an inversion technique (Kotikov et al., 1987).

The Finnish Meteorological Institute (FMI) all-sky camera (ASC) in Longyearbyen (LYR, $78.20^{\circ} \mathrm{N}, 15.70^{\circ} \mathrm{E}$ ) monitored auroras providing one frame every $20 \mathrm{~s}$. The all-sky camera operates in the $557.7-\mathrm{nm}$ emission line. Corrected geomagnetic coordinates (CGM) of the camera are $75.33^{\circ} \mathrm{N}$, $111.52^{\circ} \mathrm{E}$.

The interplanetary conditions were obtained from two satellites, IMP-8 located at $[\mathrm{X}, \mathrm{Y}, \mathrm{Z}] \mathrm{GSM}=[12.5,-33.7$, $-6.8] R_{E}$, and Wind located at [X, Y, Z] GSM=[43.4, -62.0, 8.4] $R_{E}$. Both satellites provided interplanetary magnetic field (IMF) data. The solar wind velocity and dynamic pressure (measured by Wind) were fairly stable at $360 \mathrm{~km} \mathrm{~s}^{-1}$ and $1.7 \mathrm{nPa}$, respectively. By comparing IMF disturbances (variations) observed by the two satellites, the propagation time from IMP-8 to Wind was determined to be 5 min (i.e. Wind observed the same IMF features that were observed at the IMP-8 location $5 \mathrm{~min}$ earlier). Simple calculations show that in the $\mathrm{X}-\mathrm{Y}$ plane the solar wind features were

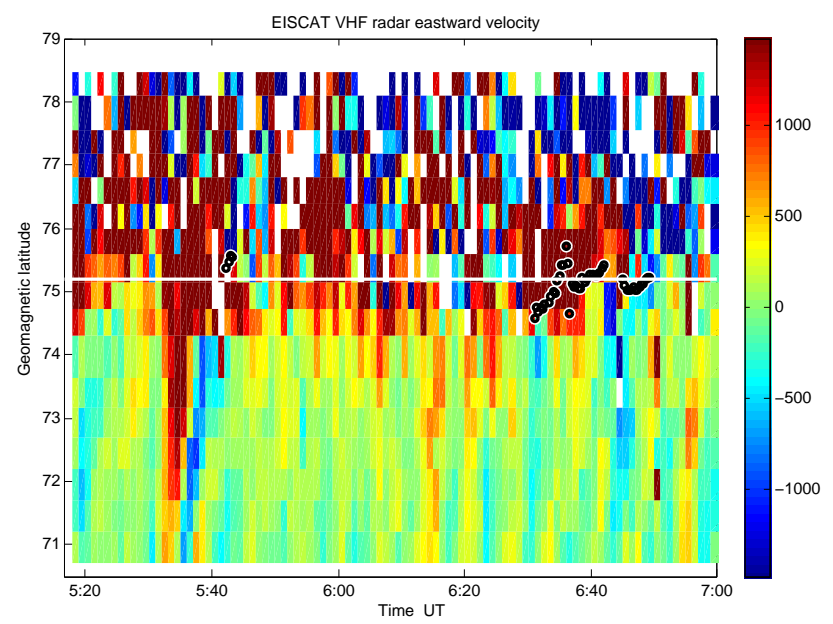

Fig. 1. East-west flow estimated from beam-swing analysis of the EISCAT CP4 split beam data $\left[\mathrm{ms}^{-1}\right]$ (see text for details). Circles indicate latitude of the arcs discussed in the paper. The horizontal white line indicates the latitude of the EISCAT Svalbard radar.

stretched at an angle of $30^{\circ}$ to the Earth-Sun line. Hence, the solar wind parameters at the Earth's bow shock (assumed at $14.6 R_{E}$ ) corresponded to those measured 21 and $26 \mathrm{~min}$ later by IMP-8 and Wind satellites, respectively. Transition from the bow shock to the ionosphere takes $7 \mathrm{~min}$, including $5 \mathrm{~min}$ for passing the magnetosheath to the subsolar magnetopause and 2-min Alfvén transit time to reach the near-noon ionosphere (Lockwood et al., 1989). Taking into account the above calculations, data from the IMP-8 satellite should be time-shifted by 14 min to show the expected time of the IMF impact in the near-noon polar cap ionosphere.

Finally, global ultraviolet images of the auroral oval obtained from the Visible Imaging System (VIS) on board the Polar satellite (Frank et al., 1995) were used to monitor the large-scale auroral background.

\section{Observations}

\subsection{Convection and general ionospheric background condi- tions}

The convection was estimated using EISCAT CP4 data and IMAGE magnetometer chain data. The standard "beamswing" analysis of CP4 split-beam data assumes longitudinal homogeneity on the spatial scale of the separation of the two beams. If this assumption is not valid, the east-west drift may be exaggerated in the standard analysis. Keeping this uncertainty in mind we show the CP4 derived east-west flow in Fig. 1, together with estimates of the latitude of the optical aurora which will be discussed in more detail in Sect. 3.4.

The ESR ion temperature enhancements also confirm the presence of strong ion flows at the periods when such are indicated by the CP4 data, but that the velocity never exceeds $2 \mathrm{kms}^{-1}$, as indicated by the standard analysis CP4 data. We will show in a later section currents in the east-west 


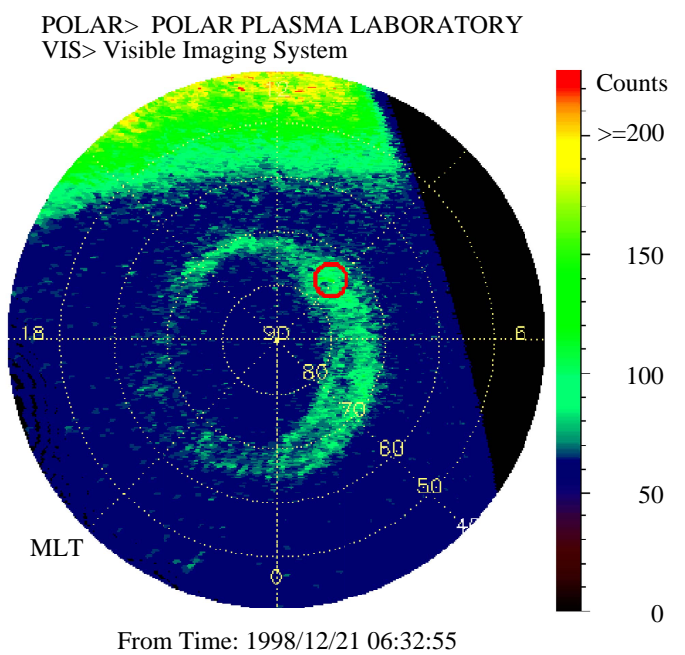

Fig. 2. Polar VIS ultraviolet image of the auroral oval in magnetic latitude and magnetic local time coordinates. The field-ofview of the Longyearbyen all-sky camera at the indicated time (06:32:55 UT) is shown by the red circle.

direction derived from IMAGE magnetometer chain X- and Z-components (using the inversion technique of Kotikov et al., 1987), together with all-sky camera keograms. From Fig. 1 we can see that the ESR was making observations in the vicinity of a border between the eastward flow on the poleward side and the low flow (corotation) with significant Pc 5 pulsations on the equatorward side. Polar VIS data shows that the observations were made inside the main UV auroral oval, as shown in Fig. 2.

Finally, we notice that the measurements were obtained during the polar night, with the solar zenith angle at Longyearbyen being in the range from $113.2^{\circ}$ at $05: 00 \mathrm{UT}$ to $107.3^{\circ}$ at $07: 00 \mathrm{UT}$.

\subsection{Overview of EISCAT Svalbard radar data}

Data from the EISCAT Svalbard radar, as well as the luminosity in the all-sky images which correspond to Longyearbyen, are collected in Fig. 3. Also shown are the IMF data from the IMP-8 satellite. As can be seen from panel (a) of this Figure the interplanetary magnetic field was dominated by the $B_{y}$ component, with $B_{z}$ mostly weakly negative until about 06:10 UT. The electron density measured by the ESR is shown in panel (b) of Fig. 3. Three features are of importance for this study:

1. The presence of significant electron densities at Eregion altitude throughout most of the period, with a peak density at around $115 \mathrm{~km}$. Considering the fact that the measurements were made in ionospheric darkness, this indicates precipitation, most likely precipitation of electrons with an energy up to about $5 \mathrm{keV}$ (Rees, 1989; Kirkwood and Osepian, 1995). An inver-

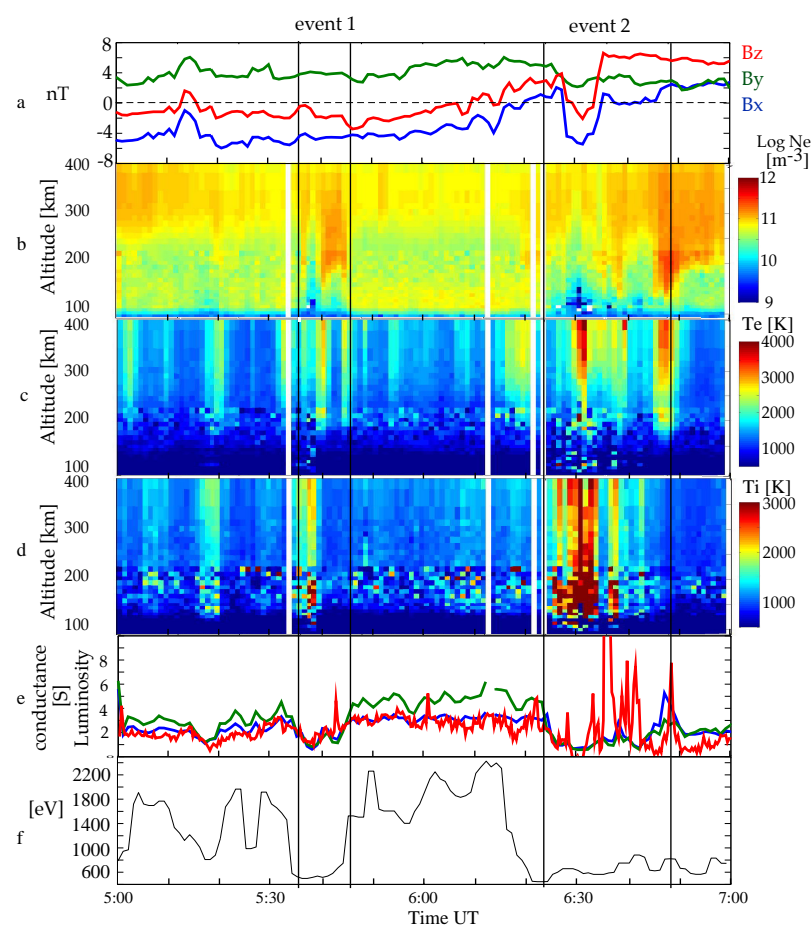

Fig. 3. (a) IMP-8 Interplanetary magnetic field data, (b), (c) and (d) EISCAT Svalbard radar (ESR) electron density $\left[\mathrm{m}^{-3}\right]$, electron and ion temperature estimates $[K]$ (e) Hall (green line) and Pedersen (blue) conductances $[S]$ calculated from ESR and corresponding line-of-sight integrated luminosity (arbitrary scale, red line) from the FMI Longyearbyen all-sky camera, (f) average energy of precipitating electrons $[\mathrm{eV}]$ derived from EISCAT ionization profile.

sion technique can be used to obtain the source energy spectrum from the ESR data (Sect. 3.3).

2. There are several structures with enhanced electron density in the upper E-region and lower F-region which appear quite typical for dayside auroral arcs, as previously reported in Watermann et al. (1993); Nilsson and Andersson (1997); Nilsson et al. (1998). The peak energy of this precipitation appears to be about $1 \mathrm{keV}$, judging from the ionization altitude interval (see also Sect. 3.3).

3. There are some regions with very low electron density in the time periods just adjacent to the arc observations. This could be a phenomenon related to high electric fields, cavity formation in the return current region just adjacent to the arcs, or just an absence of the more energetic precipitation (feature 1 above). Two periods show clear, low-density regions, arcs, and (as will be discussed later), the strongest electron and ion temperature enhancements. These are marked in Fig. 3 as event 1 (low-density region centered around 05:37 UT) and event 2 (low-density region around 06:30 UT).

From the incoherent scatter radar data we have calculated the Hall and Pedersen conductances (Fig. 3e, green and blue lines, respectively). We have used the MSIS90 model 
(Hedin, 1991) and assumed equal electron, ion and neutral temperatures in the calculation of conductance (see Senior, 1991, and references therein for details on the calculation of conductance from ISR data). The Pedersen conductance in the diffuse precipitation region is $3.2 \pm 0.6 \mathrm{~S}$, where the indicated uncertainty is the variance in the estimate over the observation interval. The uncertainty in each estimate (based on the uncertainty estimate from the EISCAT analysis) is significantly smaller. In the arcs of events 1 and 2 the conductance is about 2 to $3 \mathrm{~S}$. The highest conductance observed is for an arc observed at the end of event 2 (at 06:48 UT), with a Pedersen conductance of $5 \mathrm{~S}$. The lowest conductance observed was $0.5 \mathrm{~S}$, and the conductance observed in the low-density regions were in the range $0.7 \pm 0.2 \mathrm{~S}$.

The conductance enhancements (a signature of precipitation) are compared with an estimate of the luminosity in Fig. 3e. Ten pixels from the keogram around the location of the ESR field-of-view at $150 \mathrm{~km}$ were added to obtain an ESR site luminosity estimate (Fig. 3e, red line). It is not obvious that this luminosity in "arbitrary units" should show anything but a rough morphological similarity to the ESR measurements. Furthermore, there are indications that some clouds were present which may provide scattered light which appear as diffuse aurora. However, as can be seen in Fig. 3, panel (e), the conductance and luminosity follow each other closely in the regions of harder precipitation. Indeed this is the theoretically expected behavior if the flux of electrons is modulated while the characteristic energy remains constant.

We have also performed calculations of the expected variance of the luminosity based on the EISCAT density profiles (see Sect. 3.3, confirming that such close covariation can be expected for the current conditions). It is thus clear that the low-density regions (in particular the main low-density regions in the beginning of cases 1 and 2) correspond to very low luminosity, indicating that the absence of precipitation is a major factor behind the appearance of these E-region low-density structures. An alternative possibility could be cavity formation in a downward current region against the background of diffuse precipitation, which would yield low density without a corresponding decrease of the luminosity.

The F-region electron temperature is enhanced on a number of occasions throughout the studied period, as can be seen in Fig. 3, panel (c). There are sporadic electron temperature enhancements throughout the observation period. Whereas some arcs as identified from E-region density enhancements also show F-region electron temperature enhancements, there is no one-to-one correspondence. Often there is no obvious E-region density enhancement associated with the electron temperature increase. This is similar to observations in the cusp, and a likely cause is a significant flux of soft electron precipitation, typically around $100 \mathrm{eV}$. Such precipitation may thus be present to a varying degree in all the regions observed.

The ion temperature is enhanced on a number of occasions just as the electron temperature. As can be seen in panel (d) of Fig. 3, temperatures of $4000 \mathrm{~K}$ are observed, and even higher in some cases. This corresponds to an ion velocity of roughly $2 \mathrm{kms}^{-1}$, using the modeling work of Schunk et al. (1975). The EISCAT ion temperatures are somewhat noisy and there may be reason to doubt the very highest temperature estimates. The uncertainty estimates from the EISCAT analysis are small, but temporal and/or spatial change of the ion drift on time scales shorter than the analysis preintegration/smaller than the radar beam can yield a broadening of the EISCAT spectra and thus an apparent increase in the ion temperature. However, the high ion temperatures are nevertheless a good indicator of strong or moderately strong and variable electric fields. By comparison of panels (b) and (d) of Fig. 3, it can be seen that the strongest ion temperature enhancements are seen in the regions with low density, adjacent to arc structures.

\subsection{Energy spectra derived from EISCAT data}

The atmospheric ionization altitude profile resulting from energetic particle precipitation is energy- and pitch-angle dependent. Briefly, more energetic particles can penetrate to lower altitudes (Rees, 1989). Measurements of ionospheric density profiles can therefore be inverted, to obtain the source distribution, giving rise to the observed ionospheric electron density distribution (assuming steady state or using observed temporal variability) (e.g. Kirkwood and Osepian, 1995). We have used a similar calculation based on the algorithm described in Sergienko and Ivanov (1996), solving the linear system of equations in an iterative fashion. A large number of Maxwellian distributions were assumed in the final calculation rather than the typical mono-energetic beams. In this way a smooth energy spectrum is achieved. It is assumed that all ionization is produced by electrons, as the energy flux of ions is typically much smaller. The result of the energy spectra calculations is summarized in Fig. 4, which shows three energy spectra from the structured precipitation observed at 05:40 (blue), 06:37 (blue bold) and 06:45 UT (blue dotted) in the ESR data. The green and red lines show sample energy spectra of the diffuse, harder precipitation, from 05:25 and 06:02 UT, respectively. It is clear that the arcs all have similar source spectra, and that the energy is significantly lower than for the diffuse precipitation. The diffuse precipitation has an average energy of about $2 \mathrm{keV}$. The more energetic part is clearly consistent with a central plasma sheet (CPS) source. The arcs are created by a colder electron population, with an average energy of about $500 \mathrm{eV}$ (calculated for energies above $200 \mathrm{eV}$ ), consistent with a boundary plasma sheet (BPS) or low-latitude boundary layer (LLBL) source. However, this population may also be even colder (i.e. ionospheric) with electrons accelerated by a parallel potential drop, as can be expected for discrete aurora. These ESR measurements do not have sufficient resolution to distinguish between these two possibilities. Panel (f) of Fig. 3 shows the average energy as a function of time.

The energy input can also be determined, and is about $0.5 \mathrm{erg} \mathrm{cm}^{-2} \mathrm{~s}^{-1}$ for the diffuse precipitation and 1$3 \mathrm{erg} \mathrm{cm}^{-2} \mathrm{~s}^{-1}$ for the arcs studied in detail. Using the model of the auroral green line (Ivanov et al., 1993) and the electron 


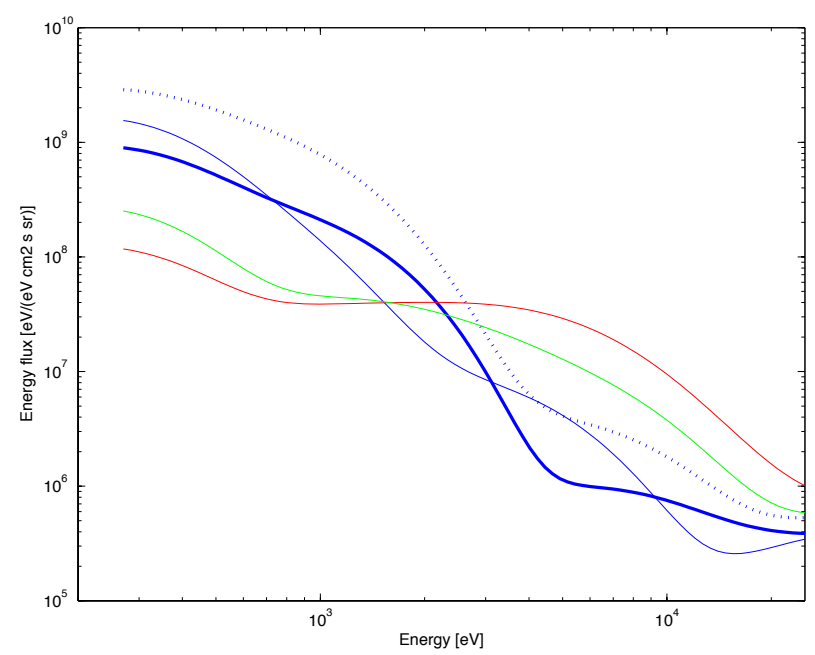

Fig. 4. Energy spectra of the source populations giving rise to structured precipitation at 05:40 (blue line), 06:37 (bold blue) and 06:45 (dotted blue) UT and diffuse precipitation (sample from 05:25 UT, green line and 06:02 UT, red line).

spectra derived from the EISCAT data as input parameters of the model, we calculated the 557.7-nm emission intensity. The calculations showed that in the diffuse precipitation region the conductance and luminosity should indeed vary together in the way shown in panel (e) of Fig. 3. Furthermore, the altitude of maximum emission was determined to be $115 \mathrm{~km}$ for the diffuse precipitation.

\subsection{Overview of all-sky camera data}

The all-sky camera data for the period between 05:00 and 07:00 UT is summarized using a keogram along the magnetic meridian, shown in Fig. 5 (arbitrary units). The latitude scale (y-axis) assumes an emission altitude for the diffuse precipitation of $115 \mathrm{~km}$, determined from simulations of the expected emissions based on ESR measurements (see Sect. 3.3). The structured precipitation occurs at higher altitude, about $150 \mathrm{~km}$, as determined from ESR measurements in the same way, which is also consistent with typical morning structured aurora, as determined by Starkov (1968); Jack and Hallinan (1994). For this study the structured aurora of interest occur close to zenith, so that the altitude assumption is not very important.

Superimposed on the keogram are contour lines of the ionospheric Hall current (green lines, eastward current; yellow lines, westward current, $\mathrm{A} / \mathrm{km}$ ). The zero line is shown with a thick yellow line. A thick red line at the latitude of ESR indicates the times when ESR observed the more energetic precipitation of CPS-like character. The times of events 1 and 2 are indicated by vertical red lines. The Hall current estimates show that the large-scale convection was eastward, consistent with the standard interpretation of the CP4 data.

For event 1 the most important feature to note is the general decrease in the diffuse luminosity, which was also seen in the estimate of the luminosity over ESR shown in Fig. 3,

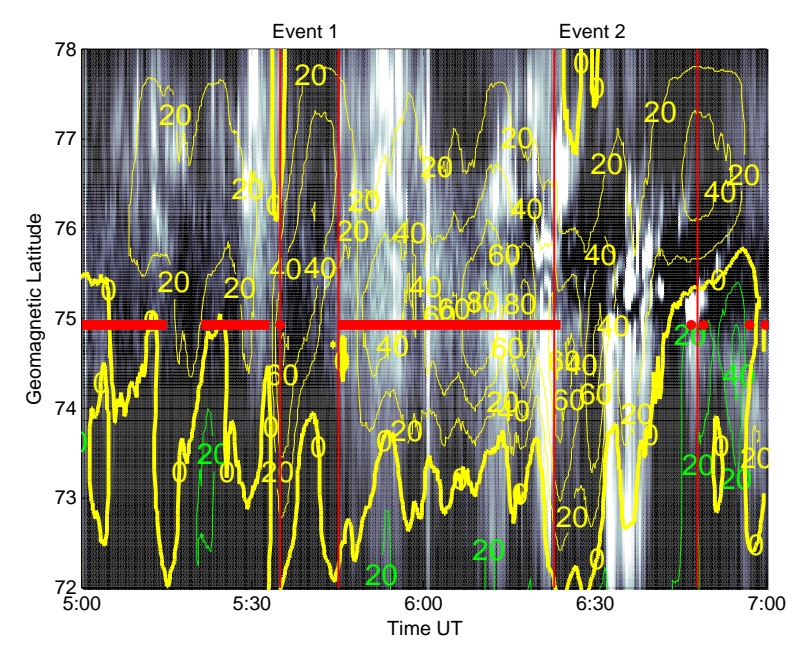

Fig. 5. Keograms along the magnetic meridian. The colour scale is in arbitrary units. Contour lines show ionospheric Hall current, $\mathrm{A} / \mathrm{km}$. Green contour lines show the eastward current and yellow contour-lines the westward current. The zero line is shown with a thick yellow line. A thick red line at the latitude of ESR indicates the times when ESR observed the more energetic precipitation of CPS-like character. Vertical red lines indicate the times of events 1 and 2. The y-axis shows geomagnetic latitude.

panel (e). This confirms that the low-density region observed by ESR corresponds to low luminosity. It also shows that the low density is not a result of the luminosity region moving away from the radar (at least not with a convection speed in the latitudinal direction), but rather a larger scale modulation of the diffuse precipitation. We are also able to identify some structured precipitation occurring during the absence of the large-scale diffuse precipitation, intensifying at and after the time of minimum diffuse precipitation. Event 1 corresponds to a pulse of strong east-west flows and currents being initiated over a large latitude range, approximately simultaneously. This, and the longer periodicity of the pulsations at higher latitudes, is consistent with field-line, eigen-mode, toroidal oscillations.

For event 2 the first thing to note from Fig. 5 is the lowluminosity region observed around 06:28-06:29 UT, coincident with enhanced current. This is somewhat similar to event 1 and indeed corresponds to the lowest density region observed in the ESR data. The luminosity was very low throughout the all-sky camera field-of-view. Just after 06:30 UT low luminosity and density is found in a narrow region between two arcs. The equatormost of these arcs is located at the boundary between strong convection and the low flow/corotation region (as will be discussed later, the current shown in Fig. 5 extends equatorward of this). This is consistent with the upward field-aligned current expected at this shielding boundary. The strong convection is estimated to be eastward by the somewhat uncertain CP4 estimates, and this is indeed consistent with the magnetometer-derived Hall current estimates. 

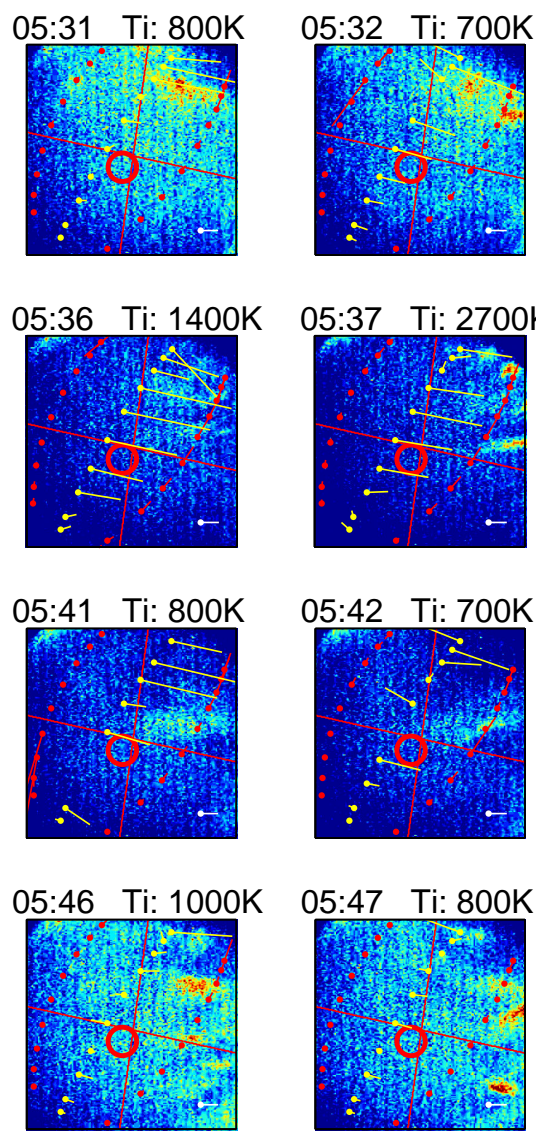
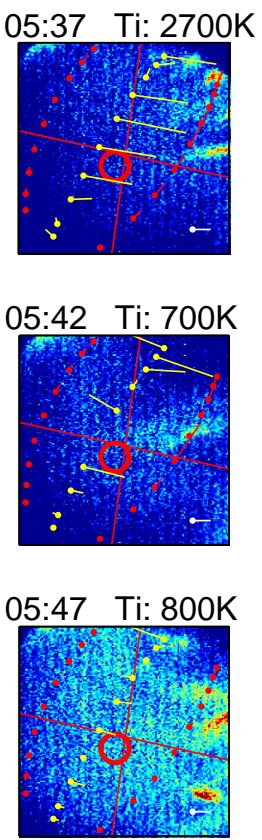
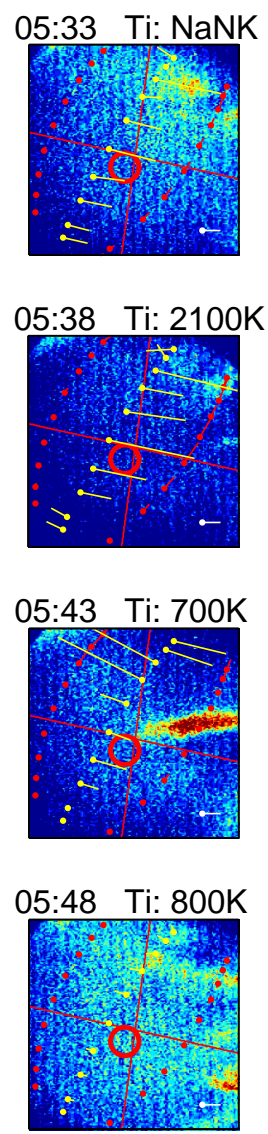
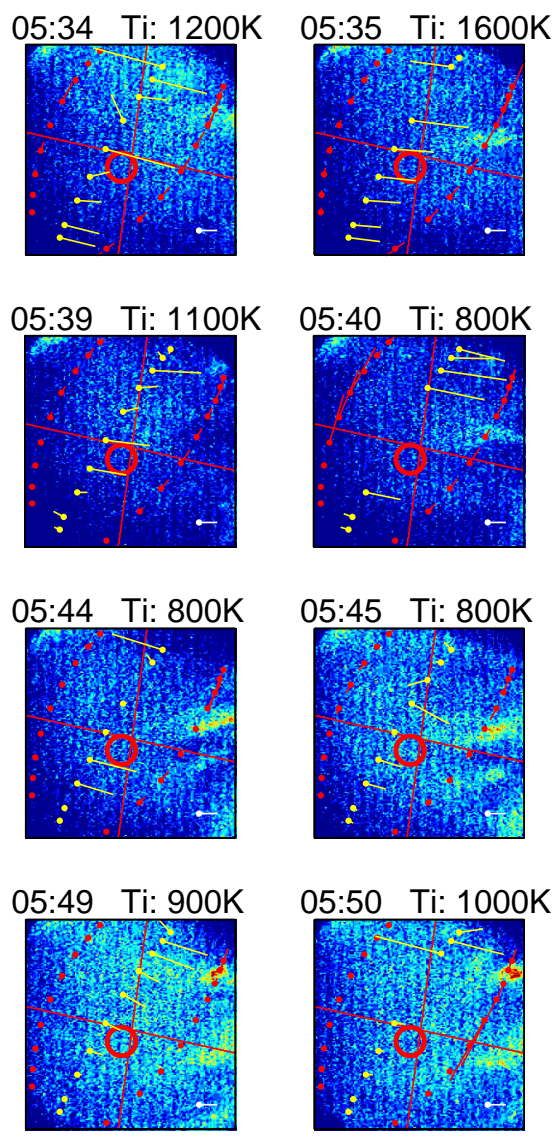

Fig. 6. All-sky images integrated to 1-min time resolution. The $\mathrm{x}$ and $\mathrm{y}$ axis show pixel number. The ESR field-of-view at $150 \mathrm{~km}$ is in the center of the red circle. Magnetic meridional and zonal lines are indicated by red lines. Yellow lines starting from a dot indicate the EISCAT $\mathrm{CP} 4$ beam-swing derived velocity vectors. The CP4 line-of-sight velocities are shown as red lines originating from dots along the radar beam projection. A white line starting from a dot indicates the length corresponding to $1 \mathrm{~km} / \mathrm{s}$. The time of observation and ESR ion temperature are indicated above each image.

For the two detailed cases we show sequences of all-sky frames in Figs. 6 and 7. Only the central part of each image is shown, together with a circle centered on the expected location of the ESR field-of-view at $150 \mathrm{~km}$ altitude, the magnetic meridional and zonal lines, the line of sight velocities of the EISCAT VHF CP4 experiment, and the estimated convection from the standard analysis of the CP4 beam-swing data. Note that this is likely to be an overestimation of the real convection (see Sect. 3.1). The all-sky images have been integrated to obtain the same time resolution as the ESR and CP4 data (1 min). Time and corresponding ion temperature from ESR are shown above each image. The ion temperature is an average over the 120 to $190 \mathrm{~km}$ altitude range. The time follows the EISCAT convention and marks the end of the integration interval.

\subsection{Detailed observations, event 1}

Figure 6 shows a sequence of all-sky images from event 1, starting at 05:30 UT. There is initially a large-scale patch of diffuse luminosity which possibly fades to west and south (panel (1)). Some structured luminosity, enhanced com- pared to the background, is also seen, at least from panel (5). The large-scale diffuse luminosity then fades over the entire field-of-view, with no discernible motion, and remains low throughout panels (6) to (12). The agreement between the low luminosity over ESR from these images and the low E-region plasma density measured by ESR gives confidence that this is indeed mainly due to a large-scale modulation of the diffuse precipitation, and not to clouds. The ESR ion temperature estimates show that the ion temperature, and thus the electric field, increased from panel (4) (05:34 UT), reached a peak of $2700 \mathrm{~K}$ at $05: 37$ (panel (7)) and then returned to the background level at 05:40 (panel (10)). This is consistent with (1) the CP4 data which shows increased electric field and with (2) the magnetometer data which show an increased current for this time. Notable is that the low luminosity reasonably indicates low conductance, but the current increases anyway, further consistent with a strong enhancement of the electric field (though probably not as strong as is indicated by the CP4 beam-swing data). After this a patch of structured precipitation intensifies just east and north of ESR (panel (13)), which must be the same structure as was seen in the ESR data at 05:40 UT, though it is barely discernible 

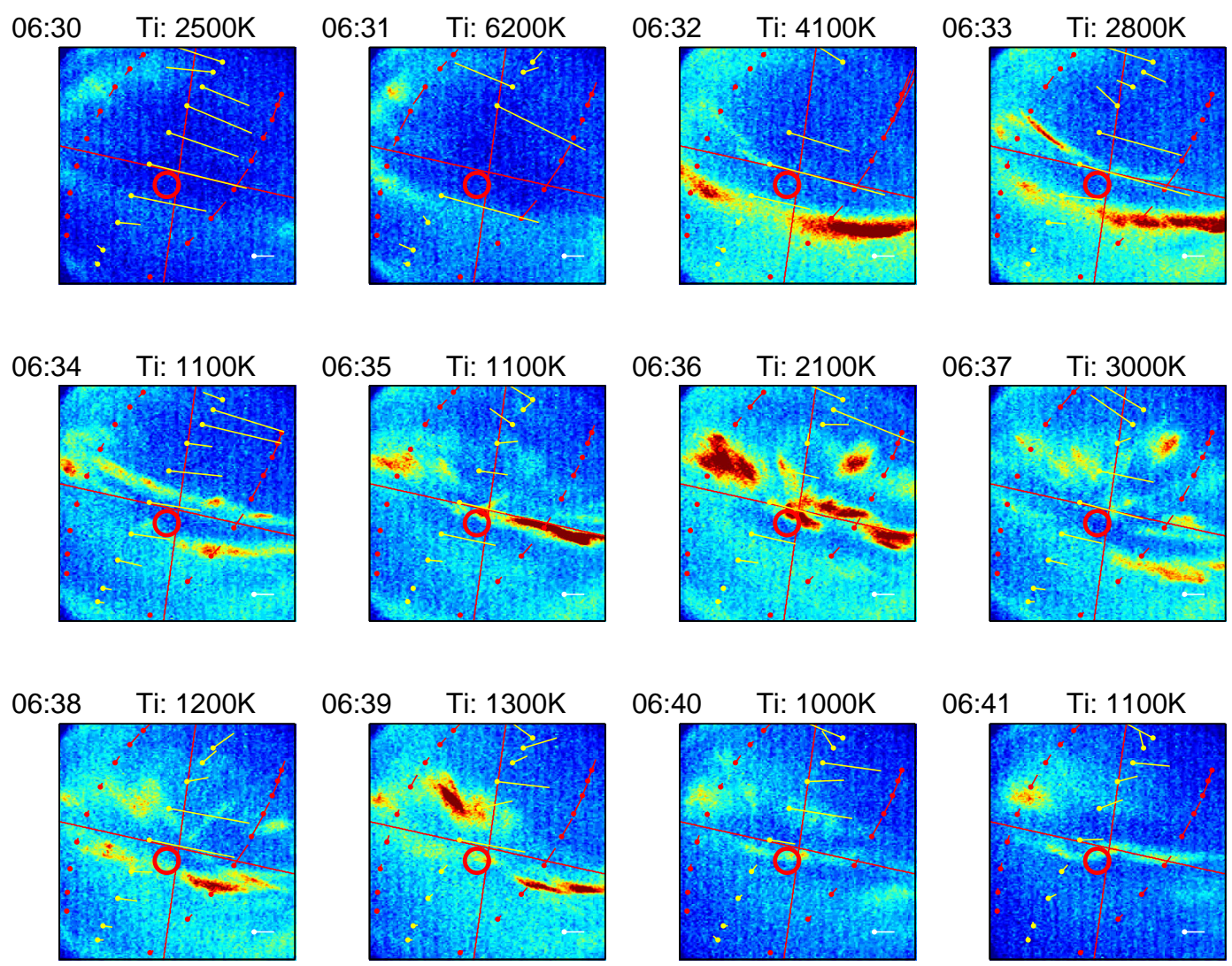

Fig. 7. All-sky images integrated to 1-min time resolution. The $\mathrm{x}$ and $\mathrm{y}$ axis show pixel number. The ESR field-of-view at $150 \mathrm{~km}$ is in the center of the red circle. Magnetic meridional and zonal lines are indicated by red lines. Yellow lines starting from a dot indicate the EISCAT CP4 beam-swing derived velocity vectors. The CP4 line-of-sight velocities are shown as red lines originating from dots along the radar beam projection. A white line starting from a dot indicates the length corresponding to $1 \mathrm{~km} / \mathrm{s}$. The time of observation and ESR ion temperature are indicated above each image.

in the all-sky image (panel (10)). After this the diffuse precipitation re-intensifies (panels (15) and onward), both north and south of the structured luminosity. This is noteworthy because the structured precipitation has a different source population compared to the diffuse precipitation (according to the EISCAT-derived energy spectra of the source population) while it occurs inside the region of diffuse precipitation. Finally, we note that whereas the strongest structured luminosity (panel (13)) occurred after the intense electric fields and the decrease in diffuse luminosity, there is considerable structure within the diffuse background precipitation during and after "recovery" (panels (15) to (20)).

\subsection{Detailed observations, event 2}

Figure 7 shows a sequence of all-sky images from event 2, starting at 06:31 UT. The ion temperature is enhanced just poleward of the arc which forms (panels (1) and (2)), then reduces somewhat as the arc intensifies and a new arc forms poleward of ESR and presumably poleward of the flow channel (panels (3) and (4)). After this (panels (5) and (6)) lower ion temperatures are observed as the equatorward arc fades. Ion temperatures increase again when the equatorward arc re-intensifies (panels (7) and (8)). Finally, the equatormost arc moves poleward past the radar site and we again observe lower ion temperatures. The data are fully consistent with a region of enhanced perpendicular electric field on the poleward side of the equatormost arc, consistent with the direction of the background convection (implied by the CP4 convection and magnetometer data). The equatormost arc clearly occurs at the equatorward border of the strong flow, i.e. at the shielding boundary. If the flow was indeed eastward, this would be consistent with an upward field-aligned current in the same region. The luminosity is also clearly enhanced over a large area equatorward of the equatormost arc, consistent with a simple picture of CPS diffuse precipitation occurring only equatorward of the arcs and strong flow.

\section{Discussion}

\subsection{Relative location of observations}

The first question to answer when discussing the location of dayside aurora is whether it occurs on open or closed fieldlines. The arcs reported here (i.e. those seen both in radar and ASC data) occur in an eastward flow region in the morning 
sector or on the border towards a corotating (shielded) region. This strongly indicates closed field-lines. The precipitation, giving rise to the diffuse luminosity and corresponding ionospheric density enhancement, is clearly of CPS origin. This is in turn consistent with the statistical results concerning apparent source regions of dayside precipitation of Newell and Meng (1992). The pulsations (Pc5) seen equatorward of the eastward flow region and aurora (in CP4 and magnetometer data) are another possible indicator of magnetospheric regions. The latitude above where no Pc5 pulsations are seen could be the open/closed field-line boundary, but this is not consistent with the other data. The near-Earth current sheet is another geophysical border which may limit field-line oscillations poleward of its ionospheric projection. It is also possible that the field-line oscillations are excited by the periodic flow and auroral intensification, and thus occur just equatorward of the flow region/auroral oval. Indeed Pilipenko et al. (2001) have reported a coupling between the electrojet and Pc5 intensity.

The energy spectra of the arcs/structured precipitation suggest that the source population is associated with the BPS or LLBL, or is caused by an even colder but accelerated population, whereas the diffuse precipitation is related to the CPS. As the precipitation occurs in the return flow region, BPS is more likely to be a source than LLBL. If the arcs/structured precipitation are related to the BPS/LLBL, then they should be observed poleward of the diffuse precipitation, which agrees fairly well with optical observations for event 2 . However, the structured precipitation observed during event 1 appears to occur within the spatial region where the diffuse precipitation of CPS origin occurs in a modulated fashion. The EISCAT-derived spectra can be interpreted as being a mixture of two populations, where the low-energy population dominates strongly in the structured precipitation, which points further towards an accelerated cold (ionospheric) population dominating when the CPS precipitation is inhibited.

The shielding (i.e. region 2) current is upward in the morning sector, so that the situation may be similar to that reported by Milan et al. (1999), where post-noon periodic aurora was attributed to boundary plasma sheet precipitation within the region 1 field-aligned current (which is upward post noon). In that study the periodicity of the aurora was attributed to ULF wave activity. For our case the time scale of auroral intensification is 3 to 5 min whereas the Pc5 pulsations observed equatorward of the aurora have a period of approximately $10 \mathrm{~min}$. The causal relationship may thus be that periodic intensification of the electric field and aurora at the convection border (or the magnetospheric process behind them) drive the pulsations, but the latter are strong only at resonant frequencies, not at all frequencies represented by the driving mechanism.

Finally, we note that the IMF conditions during event 1 and until 06:10 UT ( $\left.B_{z}<0, B_{y}>0\right)$ correspond to the situation depicted in Fig. 1c of Sandholt's arc classification (Sandholt et al., 1998). The diffuse precipitation (their type 3) is always present in the equatormost part. Both events 1 and 2 are related to aurora just poleward of the diffuse (type 3) au- rora, which is a type 6 aurora in the southward IMF case. The shielding arc presented here (event 2) is clearly their type 6 aurora located just poleward of the diffuse precipitation. The picture thus agrees well with that of Sandholt et al. (1998), though the type 6 aurora extends all the way to 10 MLT (07:00 UT) and the latitude is higher than usual $\left(75^{\circ}\right.$ rather than $72^{\circ}$ magnetic latitude) in our case. However, during $06: 10$ to $06: 35$ UT a transition took place from southward IMF to northward about $6 \mathrm{nT}$, according to our estimate of the delay between solar wind observations and the ionosphere. Despite this, the direction and location of convection, as well as the morphology of the aurora, is in perfect agreement with the type 6 aurora as it is expected for southward or approximately zero IMF $B_{z}$. We can conclude that in our case the type 6 aurora is related to the shielding boundary and thus to the region 2 current. Such aurora should be affected by substorm processes and be related to a CPS /BPS source, just as noted by Sandholt et al. (1998) for the type 6 aurora.

Finally, the more dynamic aurora poleward of the shielding arc might be poleward-moving auroral forms coming from the near noon MLT sector in the arc classification picture. After 06:35 UT the poleward arcs should be classified as type 4 aurora, possibly corresponding to sun-aligned arcs according to Sandholt et al. (1998). As we have no ESR observations in the vicinity of these auroras, we cannot investigate the associated current system.

\subsection{Low-density regions}

The E-region low-density regions are coincident with very low luminosity. Absence of diffuse precipitation is thus the first and simplest explanation of the low-density regions observed adjacent to arcs in the ISR data. We can thus conclude that downward currents do not cause cavities against the background of the diffuse precipitation, in the way intense downward currents caused cavities against the solar EUV produced background E-region densities in the case studied by Nilsson et al. (1998). There may still be cavity formation, but because the background conductance (in the absence of precipitation) is so low, this will probably not significantly affect the conductance on the spatial scale studied. There may be smaller structures which cannot be resolved by the radar. Studying the E-region density profiles in detail (not shown) does not show a pronounced minimum at the altitude of peak Pedersen conductance, as predicted by theories (Doe et al., 1995) and observed by Nilsson et al. (1998), and thus we cannot find clear evidence for any cavity formation.

In principle, the downward current region may inhibit the diffuse precipitation through, for example, a field-aligned potential drop accelerating electrons away from the ionosphere, and the low-conductance region could then still be a signature of a downward current region. Lacking data from the auroral acceleration region, we cannot investigate this possibility with the data set used in this study.

For event 1 it is clear that the low density and luminosity region precedes the appearance of structured precipitation. For case 2 low E-region densities are observed prior to arc 
intensification. Thus, arcs and strong electric fields appear in the regions where the diffuse precipitation is absent. This is why the low-density regions are observed just adjacent to the arcs in the ESR data. For event 1 the luminosity corresponding to this diffuse precipitation changes over most of the all-sky frame simultaneously so that it is clearly a largescale modulation of the diffuse precipitation, not the convective motion bringing a new region into the ESR field-of-view. The ESR data show clear low-density regions just adjacent to arcs / structured precipitation. At least for event 1, a straightforward explanation is that the low conductance is favorable for arc formation. The actual conductance changes measured (lowest background conductance 0.5 mho, high background conductance $3 \mathrm{mho}$ ) are in quantitative agreement with the ionospheric feedback model results of Pokhotelov et al. (2002b). Pokhotelov et al. (2002b) performed a twodimensional simulation in a dipole magnetic field geometry, including ionospheric feedback and shear Alfvén wave dynamics in the magnetosphere. It was concluded that ionospheric feedback could lead to narrow latitudinally-striated auroral structures, in particular if background conductance was low enough and magnetospheric energy input (background convection) was high enough. A background conductance of 0.5 mho was used in the simulations as low conductance and 3 mho as a conductance high enough to inhibit the arc formation.

For event 2 it may also be that the low conductance / high electric field corresponds to a different magnetospheric region (LLBL / BPS) and that the aurora is forming on the border between these regions. This interpretation is further supported by Fig. 5, where one can see the border between the equatorward diffuse luminosity and a low luminosity region moving equatorward from 06:00 UT, when this boundary is located at the top of the figure. At the time of event 2 this border is close to ESR, and the studied arc occurs on the border. However it is still the case that the low density region precedes the arc intensification rather than the opposite which would be expected from cavity formation. Furthermore there is enhanced magnetospheric input (enhanced currents) also equatorward of the shielding arc (evident from Fig. 5), but strong electric fields and the arc occur only poleward of the diffuse precipitation, further indicating the importance of the background conductance.

\subsection{Auroral arcs and associated electric fields}

The structured precipitation of event 1 is associated with a field-line eigen-mode toroidal oscillation. The observed shear is clearly consistent with an intense upward current. According to the discussion in the previous section the structured precipitation occurs in the upward current region only when the diffuse precipitation particles are not available as charge carriers, and further structuring due to ionospheric feedback occurs only in the case of low background conductance.

The relation between the equatormost arc of event 2 and the electric field is the same as is common on the nightside, i.e. strong transverse electric field on one side of the arc, in the same direction as the background convection and pointing toward the arc. In the post-midnight sector there will thus be an enhanced electric field on the poleward side just as in our observation, e.g. Opgenoorth et al. (1990); Aikio et al. $(1993,2002)$. This type of arc is normally associated with a strong upward current in the arc and a downward current in the enhanced electric field region adjacent to the arc. The current system of these arcs is thus the same as is common for nightside arcs in the same current (R2)/convection (post midnight return flow) region. It is thus reasonable to regard the arcs as part of the nightside auroral oval.

The strong electric field precedes the intensification of the equatorward arc, which is located at the shielding boundary. The time scale for the observed intensification of electric field and aurora is 3 to $5 \mathrm{~min}$ for event 2 . This is shorter than the observed pulsations at neighboring lower latitudes. Whereas the EISCAT data show that the electric field is much reduced at the arc-associated shielding boundary, the magnetometer data clearly show that the shielding is not complete. there is a significant current in the region of enhanced conductance equatorward of the shielding arc. The arc thus appears at the conductance border, not at the border of the magnetospheric energy input, consistent with the importance of ionospheric feedback.

\section{Conclusions}

The arcs studied in detail occur in the eastward flow region associated with the morning return flow or on the border between eastward flow and corotation. For these arcs one may note the following:

The conductance is enhanced in the arc regions compared to the plasma just adjacent to the arcs, though only weakly (rising from $0.5-1 \mathrm{~S}$ to $2-3 \mathrm{~S}$ ) for the cases occurring within the ESR field-of-view. The background conductance seen outside arc/cavity events is larger, $3 \mathrm{~S}$ for the case studied. The change of conductance and field-of-view integrated luminosity agrees very well between this background and the low-density regions (cavities) surrounding the arcs. This shows that absence of precipitation is a major factor behind the low conductance in the cavities. No evidence can thus be found for cavity formation against the background density enhancement caused by diffuse precipitation. Cavity formation may still play a role after arc formation. It is suggested that the background conductance enhancement caused by diffuse precipitation inhibits arc formation in the same way as solar-produced conductance enhancement may inhibit auroral arc formation.

The low-density regions are associated with strong electric fields and occur just adjacent to arcs. The close relationship between arc and cavity observations in the ESR data does not result from cavity formation in the downward current region associated with the arc but rather indicates that low background conductance is favorable for arc formation. 
The transverse electric field is strongly enhanced poleward of the arcs which could be studied in detail, consistent with post-midnight nightside arcs. The intensification of the arcs is associated with a channel of strongly increased transverse electric field. The strong flow is leading, and subsides as the equatormost arc intensify. The time scale for these intensifications and fadings was 3-5 min. The current calculated from magnetometer measurements increases in the low conductance high electric field regions, indicating that the strong electric fields result from magnetospheric driving rather than an ionospheric feedback. At least for one case reported here the arc on the shielding border (associated with the region 2 upward current) has the same current system as is common for nightside arcs in the same current (and convection) region.

The combination of incoherent scatter radar and optical data allows us to determine that the structured precipitation consisted of comparatively cold electrons (average energy $500 \mathrm{eV}$ calculated for particle energies above $200 \mathrm{eV}$ ). Electrons of ionospheric origin accelerated through a fieldaligned potential drop are a plausible source. The diffuse harder precipitation seen around (event 1 ) and equatorward (event 2) of the arcs had an average energy of about $2 \mathrm{keV}$. For event 2 the energy difference may be because diffuse and structured precipitation corresponds to different magnetospheric regions (CPS vs. LLBL/BPS).

Acknowledgements. Optical data for this study were obtained from the Finnish Meteorological Institute. We thank K. Kauristie at the Finnish Meteorological Institute for help in the all-sky camera data analysis. We are indebted to the Director and staff of EISCAT for operating the facility and supplying the data. EISCAT is an international association supported by Finland (SA), France (CNRS), the Federal Republic of Germany (MPG), Japan (NIPR), Sweden (VR) and the United Kingdom (PPARC). We thank R. Kuula at the Space Research group of the Oulu University for the EISCAT data analysis. Data of the Wind, IMP-8 and Polar satellites were obtained from the Coordinated Data Analysis Web (http: //cdaweb.gsfc.nasa.gov). We thank the data providers A. Szabo and R. P. Lepping (NASA GSFC) for the Wind IMF data, K. Ogilvie (NASA/GSFC) for the Wind solar wind data and L. A. Frank (the University of Iowa) for Polar VIS data. We thank the Finnish Meteorological Institute and other institutes who maintain the IMAGE magnetometer array. This study was funded by the Academy of Finland and the Research Council of Sweden. The work of A. Kotikov was supported by Kungliga Vetenskapsakademin.

Topical Editor M. Lester thanks O. Marghitu and another referee for their help in evaluating this paper.

\section{References}

Aikio, A. T., Opgenoorth, H. J., Persson, M. A. L., and Kaila, K. U.: Ground-based measurements of an arc-associated electric field, J. Atm. Terr. Phys., 55, 797-808, 1993.

Aikio, A. T., Lakkala, T., Kozlovsky, A., and Williams, P. J. S.: Electric fields and currents of stable drifting auroral arcs in the evening sector, J. Geophys. Res., 107, doi:10.129/2001JA009 172, 2002.
Banks, P. M. and Yasuhara, F.: Electric fields and conductivity in the night time E-region; A new magnetosphere-ionosphereatmosphere coupling effect, Geophys. Res. Lett., 5, 1047-1050, 1978.

Blanc, M. and Caudal, G.: The spatial distribution of magnetospheric electric fields at ionospheric altitudes: a review. 2. Theories, Ann. Geophys., 3, 27-42, 1985.

Doe, R. F., Vickrey, J. F., and Mendillo, M.: Electrodynamic model for the formation of auroral ionospheric cavities, J. Geophys. Res., 100, 9683-9696, 1995.

Fasel, G. J.: Dayside poleward moving auroral forms: A statistical study, J. Geophys. Res., 100, 11 891-11 905, 1995.

Frank, L. A., Sigwarth, J. B., Craven, J. D., Cravens, J. P., Dolan, J. S., Dvorsky, M. R., Harvey, J. D., Hardebeck, P. K., and Muller, D.: The Visible Imaging System (VIS) for the Polar spacecraft, Space Sci. Rev., 71, 297-328, 1995.

Golovchanskaya, I. V. and Maltsev, Y. P.: Interchange instability in the presence of the field-aligned current: Application to the auroral arc formation, J. Geophys. Res., 108, doi:10.1029/2002JA009 505, 2003.

Hedin, A. E.: Extension of the MSIS thermosphere model into the middle and lower atmosphere, J. Geophys. Res., 96, 1159-1172, 1991.

Ivanov, V. E., Sergienko, T. I., and Steen, A.: Modeling of the altitude distribution of green line (5577 $\AA$ ) luminosity in aurora, in Airglow and Aurora, edited by S. Leontiev, vol. 2050, SPIE, 1993.

Jack, T. M. and Hallinan, T. J.: Measurements of auroral rays from space shuttle, J. Geophys. Res., 99, 8 865-8 872, 1994.

Karlsson, T. and Marklund, G. T.: Simulations of small-scale auroral current closure in the return current region, in Physics of Plasmas, MIT Center for Theoretical Geo/Cosmo Plasma Physics, 1998.

Kirkwood, S. and Osepian, A.: Quantitative studies of energetic particle precipitation using incoherent scatter radar, J. Geomagn. Geoelectr., 47, 783-799, 1995.

Kotikov, A. L., Latov, Y. A., and Trosichev, O. A.: Structure of auroral electrojets by data from a meridional chain of magnetic stations, Geophysica, 23, 143, 1987.

Kozlovsky, A. and Kangas, J.: Motion and origin of noon highlatitude poleward moving auroral arcs on closed magnetic field lines, J. Geophys. Res., 107(2), doi:10.1029/2001JA900 145, 2002.

Kozlovsky, A., Safargaleev, J., Jussila, J., and Koustov, A.: Prenoon high-latitude auroral arcs as a manifestation of the interchange instability, Ann. Geophys., 21, 2303-2314, 2003,

SRef-ID: 1432-0576/ag/2003-21-2303.

Lockwood, M., Sandholt, P.-E., Cowley, S. W. H., and Oguti, T.: Interplanetary magnetic field control of dayside auroral activity and the transfer of momentum across the dayside magnetopause, Planet. Space Sci., 37, 1347-1365, 1989.

Lockwood, M., Denig, W. F., Farmer, A. D., Davda, V. N., Cowley, S. W. H., and Lühr, H.: Ionospheric signatures of pulsed reconnection at the Earth's magnetopause, Nature, 361, 424-428, 1993.

Lockwood, M., McCrea, I. W., Milan, S. E., Moen, J., Cerisier, J.-C., and Thorolfssen, A.: Plasma structure within polewardmoving cusp/cleft auroral transients: EISCAT Svalbard radar observations and an explanation in terms of large local time extent of events, Ann. Geophys., 18, 1027-1042, 2000,

SRef-ID: 1432-0576/ag/2000-18-1027. 
Lysak, R. and Song, Y.: Energetics of the ionospheric feedback interaction, J. Geophys. Res., 107, 10.1029/2001JA00 308, 2002.

Lysak, R. L.: Electrodynamic coupling of the magnetosphere and ionosphere, Space Sci. Rev., 52, 33-87, 1990.

McCrea, I. W., Lockwood, M., Moen, J., Pitout, F., Eglitis, P., Aylward, A. D., Cerisier, J.-C., Thorolfssen, A., and Milan, S. E.: ESR and EISCAT observations of the response of the cusp and cleft to IMF orientation changes, Ann. Geophys., 18, 1009-1026, 2000,

\section{SRef-ID: 1432-0576/ag/2000-18-1009.}

Milan, S. E., Yeoman, T. K., Lester, M., Moen, J., and Sandholt, P. E.: Post-noon two-minute period pulsating aurora and their relationship to the dayside convection pattern, Ann. Geophys., 17, 877-891, 1999,

SRef-ID: 1432-0576/ag/1999-17-877.

Moen, J., van Eyken, A. P., and Carlson, H. C.: Svalbard Radar observations of ionospheric plasma dynamics in relation to dayside auroral transients, J. Geophys. Res., 106, 21 453-21 462, 2001.

Newell, P. T. and Meng, C.-I.: Mapping the dayside ionosphere to the magnetosphere according to particle precipitation characteristics, Geophys. Res. Lett., 19, 609, 1992.

Newell, P. T., Meng, C.-I., and Lyons, K. M.: Suppression of discrete aurora by sunlight, Nature, 381, 766-767, 1996.

Nilsson, H. and Andersson, L.: Incoherent scatter radar and Freja satellite observation of dayside auroral arcs, Phys. Chem. Earth, 22, 669-674, 1997.

Nilsson, H., Kirkwood, S., Eliasson, L., Norberg, O., Clemmons, J., and Boehm, M.: The ionospheric signature of the cusp: A case study using Freja and the Sondrestrom radar, Geophys. Res. Lett., 21, 1923-1926, 1994.

Nilsson, H., Kirkwood, S., and Moretto, T.: Incoherent scatter radar observations of the cusp acceleration region and cusp fieldaligned currents, J. Geophys. Res., 103, 26 721-26 730, 1998.

Opgenoorth, H. J., Häggström, I., Williams, P. J. S., and Jones, G. O. L.: Regions of strongly enhanced perpendicular electric fields adjacent to auroral arcs, J. Atm. Terr. Phys., 52, 449-458, 1990.

Pilipenko, V. A., Watermann, J., Popov, V. A., and Papitashvili, V. O.: Relationship between auroral electrojet and Pc5 ULF waves, J. Atm. Sol. Terr. Phys., 63, 1545-1557, 2001.

Pokhotelov, D., Lotko, W., and Streltsov, A. V.: Effects of the seasonal assymetry in ionospheric Pedersen conductance on the appearance of discrete aurora, Geophys. Res. Lett., 29, 10.1029/2001GL014 010, 2002a.

Pokhotelov, D., Lotko, W., and Streltsov, A. V.: Harmonic structure of field-line eigenmodes generated by ionospheric feedback instability, J. Geophys. Res., 107, doi:10.1029/2001JA000 134, 2002 b.
Prikryl, P., MacDougall, J. W., Grant, I. F., Steele, D. P., Sofko, G. J., and Greenwald, R. A.: Observations of polar patches generated by solar wind Alfvén wave coupling to the dayside magnetosphere, Ann. Geophys., 17, 463-489, 1999,

SRef-ID: 1432-0576/ag/1999-17-463.

Rees, M. H.: Physics and Chemistry of the Upper Atmosphere, Cambridge Univ. Press, New York, 1989.

Safargaleev, V. V., Kozlovsky, A. E., Osipenko, S., and Tagirov, V. R.: Azimuthal expansion of high-latitude auroral arcs, Ann. Geophys., 21, 1793-1805, 2003,

SRef-ID: 1432-0576/ag/2003-21-1793.

Samson, J. C., Hughes, T. J., Creutzberg, F., Wallis, D., Greenwald, R. A., and Ruohoniemi, J. M.: Observations of a detached discrete arc in association with field line resonances, J. Geophys. Res., 96, 15 683-15 695, 1991.

Sandholt, P. E., Farrugia, C. J., Stauning, P., Cowley, S. W. H., and Hansen, T.: Cusp/cleft auroral forms and activities in relation to ionospheric convection: Responses to specific changes in solar wind and IMF conditions, J. Geophys. Res., 101, 5003-5020, 1996.

Sandholt, P. E., Farrugia, C. J., Moen, J., Noraberg, Ø., Lybekk, B., Sten, T., and Hansen, T.: A classification of dayside auroral forms and activities as a function of interplanetary magnetic field orientation, J. Geophys. Res., 103, 23 325-23 345, 1998.

Schunk, R. W., Raitt, W. J., and Banks, P. M.: Effect of electric fields on the daytime high-latitude $\mathrm{E}$ and $\mathrm{F}$ regions, J. Geophys. Res., 80, 3121-3130, 1975.

Senior, C.: Solar and particle contributions to auroral heightintegrated conductivities from EISCAT data: a statistical study, Ann. Geophys., 9, 449-460, 1991.

Sergienko, T. I. and Ivanov, V. E.: Deducing the auroral electron spectra from the EISCAT electron density measurements, in Proc. of the planning meeting for joint studies with Ørsted, the EISCAT and other ground based instruments, edited by H. Ranta, E. Turunen, and M. Hämäläinen, 54, Finnish Academy of Science and Letters, Sodankylä, 1996.

Starkov, G. V.: Auroral heights in the polar cap, Geomagn. Aeron. Engl. Transl., 8, 28-32, 1968.

Streltsov, A. V. and Lotko, W.: Small-scale electric fields in downward current channels, J. Geophys. Res., 107, doi:10.129/2002JA009 806, 2002.

Watermann, J., de la Beaujardière, O., and Spence, H. E.: Space time structure of the morning aurora inferred from coincident DMSP-F6,-F8 and Søndrestrøm incoherent scatter radar observations, J. Atm. Terr. Phys., 55, 1729-1739, 1993.

Watermann, J., Lummerzheim, D., de la Beaujardière, O., Newell, P. T., and Rich, F. J.: Ionospheric footprint of magnetosheathlike particle precipitation observed by an incoherent scatter radar, J. Geophys. Res., 99, 3855-3867, 1994. 